\title{
Lycium barbarum Fruit (Goji) Attenuates the Adrenal Steroid Response to an Exercise Challenge and the Feeling of Tiredness: A Randomized, Double-blind, Placebo-controlled Human Clinical Study
}

\author{
Harunobu Amagase (Corresponding author) \\ FreeLife International Inc., 4950 South $48^{\text {th }}$ Street, Phoenix, AZ 85040, USA \\ Tel: +1-602-333-4926Ｅ-mail: hamagase@freelife.com
}

Dwight M. Nance

Susan Samueli Center for Integrative Medicine

University of California at Irvine, 101 The City Drive South, Orange, CA 92868, USA

Tel: +1-714-456-2997Ｅ-mail: dnance@uci.edu

Received: February 6, 2012 Accepted: February 17, $2012 \quad$ Published: May 1, 2012

doi:10.5539/jfr.v1n2p3 URL: http://dx.doi.org/10.5539/jfr.v1n2p3

The research is financed by FreeLife International, Inc

\begin{abstract}
We examined the effects of Lycium barbarum fruit (goji) intake on general well-being in a randomized, double-blind, placebo-controlled 30-day intervention trial. Plasma levels of cortisol, dehydroepiandrosterone (DHEA), glucose, urea nitrogen (BUN) and lactic acid followed by an exercise challenge were assessed at the pre- and post-intervention. Relative to the placebo group $(n=19)$, tiredness and overall health were significantly improved in the Lycium barbarum group $(\mathrm{n}=20)$. Cortisol, DHEA and lactic acid levels were significantly increased by the exercise for the pre-intervention: However, at the post-intervention, Lycium barbarum intake significantly attenuated cortisol and DHEA levels. Lactic acid levels were comparable for both groups, and glucose and BUN levels were not altered. These results show that Lycium barbarum consumption attenuates the adrenal steroid response and reduces the feeling of tiredness.
\end{abstract}

Keywords: Lycium barbarum, Goji, Exercise, Cortisol, Dehydroepiandrosterone, Lactic acid, Tiredness, General Well-being

\section{Introduction}

Our previous randomized, double-blind, placebo-controlled human clinical studies showed that daily consumption of Lycium barbarum, in the form of fruit juice, GoChi ${ }^{\mathbb{Q}}$, significantly increased subjective feelings of general well-being and reduced fatigue (Amagase et al., 2008). Lycium barbarum consumption significantly enhanced in vivo immune functions as indicated by increased number of lymphocytes and blood concentrations of immunoglobulin G and interleukin (IL)-2 (Amagase et al., 2009a). In vivo anti-oxidant effects of Lycium barbarum include a significant increase in blood concentrations of superoxide dismutase and glutathione peroxidase and a significant reduction in lipid peroxidation (malondialdehyde) (Amagase et al., 2009b). Lycium barbarum intake has also been shown to increase metabolic rate/energy expenditure in a dose-dependent manner (Amagase et al., 2011b). Lycium barbarum juice used in these studies is standardized for its main active constituents, Lycium barbarum polysaccharide (LBP). Lycium barbarum is a Solanaceous defoliated shrubbery and has been a commonly prescribed traditional medicine in Asian countries for over 2,500 years (Amagase et al., 2008, 2011a; Bensky et al., 1993; Chang et al., 2001, 2008). Additional effects of Lycium barbarum include improved endurance, anti-agng, neuroprotection, anti-diabetic, anti-glaucoma, anti-tumor activity and cytoprotection have been reported (Amagase et al., 2011a).

Adrenal steroids, such as cortisol and prohormone, dehydroepiandrosterone (DHEA), regulate a variety of 
cardiovascular, metabolic, immunologic and homeostatic functions (American Heart Association, 2009; Buford et al., 2008; Walker, 2007). These "stress hormones" are released in response to a variety of physical, metabolic and psychological stressors. DHEA is produced primarily in the adrenal glands and is released along with cortisol in response to stress (Dillon, 2005). Exercise is a potent stimulus for cortisol release and exercise increases DHEA production (Filaire et al., 1998; Tissandier et al., 2001; Copeland et al., 2002). Thus, measurement of DHEA and cortisol levels in response to an exercise challenge may provide an index of the physiological effects of Lycium barbarum on the response to a physical stressor.

To extend the analysis of the physiological actions of Lycium barbarum and to investigate possible mechanisms of action as the first step, we examined the effects of Lycium barbarum intake on exercise-induced adrenal steroid release as well as lactic acid, glucose and blood urea nitrogen (BUN) concentrations in plasma at pre- and post-30-day-intervention trial under a randomized, double-blind, placebo-controlled manner.

\section{Materials and Methods}

\subsection{Lycium barbarum and placebo preparation}

FreeLife International Inc, located in Phoenix, Arizona, supplied a commercially available, LBP-standardized Lycium barbarum fruit (goji) juice (GoChi; Lot No. ASA07351) which was produced from fresh ripe Lycium barbarum fruit. Description and standardization procedures of the test material were previously described (Amagase et al., 2008). In brief, the yield of juice as a percentage weight of the starting fresh plant material is approximately $35 \%$. The juice was processed in an aseptic manner and kept refrigerated before use at 2 to $8{ }^{\circ} \mathrm{C}$. GoChi is standardized to contain a content of LBP equivalent to that found in at least $150 \mathrm{~g}$ of fresh fruit in 120 $\mathrm{ml}$, the amount customarily consumed in traditional Chinese medicine (Yu et al., 2007; Amagase et al., 2011a). Based on our previous dose-seeking study on energy expenditure (Amagase et al., 2011b) and other various studies (Amagase et al., 2008, 2009a, 2009b), we used $120 \mathrm{ml}$ of GoChi in the present studies as an established dose.

Placebo control material (Lot No. A198) was carefully prepared as previously described (Amagase et al., 2009a) to match the color, flavor, and taste of GoChi in a formulation of sucralose $(10 \mathrm{mg})$, artificial fruit flavor $(30 \mathrm{mg})$, citric acid $(60 \mathrm{mg})$, and caramel color $(12 \mathrm{mg})$ in $30 \mathrm{~mL}$ of purified water. It was packaged in the same type of container; however, it provided no nutritional value or LBP.

In addition, a novel trace amount of flavor was added to both active and placebo preparations to mask the differences, so no study participants had been exposed to the flavor-masked samples specifically prepared for the present study.

\subsection{Clinical study}

A 30-day intervention study was performed in a randomized, double-blind, placebo-controlled manner. To maintain high compliance with our first exercise challenge test and make the test under a similar physical activity condition in the office work, we recruited the participants from in-house. All randomized participants were healthy men and women, age $18 \mathrm{y}$ and older (average age $=33.6 \pm 1.9 \mathrm{y}$ ) (Table 1A). The CONSORT chart in Figure 1 shows the population including ethnic backgrounds. Recruitment was conducted to ensure that participants were serious about participating in these studies and well aware of its demands. All participants in the study were fully informed of the purpose of the study, and signed the Human Subjects Informed Consent forms approved by the Internal Review Board organized under the Helsinki Declaration. Exclusion and inclusion criteria were same as the previous studies (Amagase et al., 2008, 2009a, 2009b). Following enrollment, all participants completed a 2 to 4 week wash-out period during which time they discontinued use of any dietary supplements, including Lycium barbarum or Lycium barbarum-containing foods, if any, energy drinks, caffeinated beverages or tea, and these restrictions were continued throughout the study based upon the self-declaration in the daily dietary diary and verbal confirmation. Our previous studies have shown that there were no statistically significant differences after this wash-out period in various subjective indicators. A total of 39 healthy male and female adults were randomly assigned to either the Lycium barbarum treatment or placebo control group for this 30-day intervention study (Figure 1). Sixty-seven percent were women. Male and female participants were randomized separately to ensure an equal number of men and women in each treatment group. The participants, all investigators and staff involved in this study were blinded to the participant's treatment assignment. Tested products were assigned a number or letter code. This code remained unrevealed to the investigators involved in the study until after completion of the data analyses. No participants were pregnant during the study based upon standard urine pregnancy test (Kurkel Enterprises, HCG Lot No. 5-06257, Redmond, Washington). There were no statistical differences in demographic and clinical characteristics of the study population, and pre-study diet on the parameters of dietary intake (Table 1A), average Lycium barbarum 
consumption history, if any, and consumption patterns for other beverages such as sweetened beverages (soda), coffee, tea and alcoholic beverages, or smoking history. Food intake was monitored throughout the study by means of daily dietary diaries maintained by the participants. We noticed that self-reported dietary diary may not be completely accurate in the clinical study. However, we believed that it was better than no food log to trace the food records, and may reflect rough caloric intake during the study, as participants were aware of what they eat. In fact, average caloric intake was somewhat realistic based on their diary (Table 1A). All participants reported regular intake of a Western style diet. Daily energy intake was calculated by combining macronutrients intake from the individual diary recorded in the evening with their entire food, snack and beverage intake. A review of the participants' daily diary, there appears to be no change in the participants' other juice intake during the intervention period compared to pre-intervention. All participants were monitored daily to ensure full compliance with the protocol including restriction of dietary intake. Upon randomization, participants consumed $120 \mathrm{ml}$ of Lycium barbarum juice or placebo each morning shortly after a meal in front of the researcher on weekdays for a period of 30 days under free-living conditions. To monitor the weekend compliance, we recovered empty bottles on the following Monday. No dropouts occurred in either group during the intervention period. Based upon the previous studies (Amagase et al., 2008, 2009a, 2009b, 2011b), a sample size of 39 participants was deemed to be sufficient to detect effectiveness of Lycium barbarum alone with $95 \%$ confidence and $80 \%$ power.

At the pre- and post-intervention period (Day 1 or Day 30), all participants were given physical anthropometric measurements collected following an overnight $12 \mathrm{~h}$ fast and included: body weight and body mass index (BMI) (Seca 703, Hamburg, Germany) (Figure1). All participants were administered a written questionnaire with a rating scale (0-5) (Amagase et al., 2008) at the time of pre- (Day 1) and post-intervention (Day 30) immediately before the exercise challenge (Figure1). The questionnaire consisted of physical and psychological fatigue-related symptoms, such as fatigue, feelings of tiredness, musculoskeletal questions, cardiovascular questions, and questions regarding possible side effects.

To provide a comparable short and intense exercise challenge, each participant was tested on a ramp-type progressive electronically braked either upright (Schwinn, Model 126, Vancouver, WA) or recumbent cycle ergometer (Schwinn, Model 226-recumbent, Vancouver, WA) (Figure 1). This exercise challenge was performed only on Day 1 (pre-intervention) and Day 30 (post-intervention) as an acute intense physical stress. After resting on the cycle ergometer for 1 min of unloaded pedaling to confirm the resting heart rate, the work rate (WR) was increased by 20 to $30 \mathrm{watt} / \mathrm{min}$ adjusted according to the participant's age and fitness level by monitoring display of the heart rate. Workloads were individualized for each participant and were calculated to be equivalent to the WR corresponding roughly to $70 \%$ of age-adjusted maximum heart rate as determined non-invasively based on American Heart Association (2011) and Sharkey et al (2007). Participants were vigorously encouraged during the high-intensity phases of the exercise protocol equal 12 to 14 minutes (Radom-Aizik et al., 2008, 2009) until preset $200 \mathrm{kcal}$ were burned. The initial result of the exercise challenge for all the participants at the pre-intervention time point on heart rate, watts and calorie burned did not show any statistically significant differences for the post-intervention challenge as shown in Table 1B. In the background of the participants, there were no statistical differences in the average exercise frequency or length between the groups (Table 1A). Changes in plasma concentrations of DHEA, cortisol, glucose and lactic acid were assessed pre- and post-intervention immediately before and after the exercise challenge (Figure 1). Hormone concentrations were measured by enzyme-linked immunosorbent assay (ELISA) (Diagnostic Systems Laboratories, Inc. Webster, Texas), lactic acid levels determined in a YSI 2300 Stat Plus analyzer (YSI, Inc., Yellow Springs, Ohio), glucose and BUN were determined by standard medical laboratory methods (LabExpress, Phoenix, Arizona).

\subsection{Statistical analysis}

Dietary intake data were analyzed with non-parametric Mann-Whitney U-Test (placebo vs Lycium barbarum). For all clinical symptom questions, each question was graded and the scores analyzed for changes between preand post-intervention with the nonparametric Wilcoxon matched pairs tests. A 2 x (2) mixed ANOVA (group x test) was used for body weight, BMI, and a 2 x (2) x (2) mixed ANOVA (group x test $\mathrm{x}$ time) was used for plasma levels of hormones, glucose, BUN concentrations. Descriptive statistics were calculated for placebo and Lycium barbarum for all dependent measures and summarized as means \pm SEM. The data were processed using Statistica version 8 (StatSoft, Inc., Tulsa, Oklahoma). Differences were considered significant at $\mathrm{P}<0.05$.

\section{Results}

Significant differences $(\mathrm{P}<0.05)$ between pre- and post-intervention were found in the Lycium barbarum group $(\mathrm{n}=20)$ for questions in tiredness categories as shown in Table 2. The Lycium barbarum group showed 
significant reductions in feelings of tiredness after exercise, poor circulation, and overall health conditions. Compared to the pre-intervention results, more than $65 \%$ of people who consumed Lycium barbarum reported they did not feel tiredness after exercise. In contrast to the Lycium barbarum group, the placebo group $(\mathrm{n}=19)$ showed no statistically significant changes following the 30-day intervention period. There were no statistically significant changes in body weight, BMI or total body fat between the groups or pre- vs. post-intervention in both groups (Table 2).

DHEA, cortisol and lactic acid concentrations were all significantly increased in the placebo group after the exercise challenge for both pre- and post-treatment tests $(\mathrm{P}<0.05)$ (Table 3). Similarly, an increase in plasma levels of DHEA and cortisol was observed after the initial exercise challenge in the Lycium barbarum group before treatment; however, following Lycium barbarum treatment for 30 days the exercise-induced increase in DHEA and cortisol levels was significantly attenuated. The exercise-induced increase in lactic acid concentration was not changed by Lycium barbarum or placebo intake (Table 3). Blood glucose levels were comparable both pre- and post-intervention for both groups. BUN level post- intervention was increased in both groups, but no statistical difference was found after exercise (Table 3).

\section{Discussion}

The present study was the first human clinical study to show that Lycium barbarum intake significantly attenuated the increases in plasma DHEA and cortisol concentrations produced by a short and intense exercise challenge. Exercise represents a physical stress that challenges homeostasis. In response to this stressor, autonomic nervous system and the hypothalamic-pituitary-adrenal axis (HPA-axis) are known to react and to participate in the maintenance of homeostasis. This includes elevation of cortisol and cathecholamines in plasma (Mastorakos et al., 2005). The present study results suggest that consumption of Lycium barbarum may increase adaptability to a physical stressor such as exercise by either reducing production and function of glucocorticoids, or accelerating metabolism of these hormones. Some of the immunomodulatory actions of Lycium barbarum may be mediated in part through changes in the HPA-axis. Lycium barbarum intake may interact with cortisol by stimulating the immune system as reported in a previous clinical study (Amagase, 2009a). Likewise, the reported ability of Lycium barbarum to significantly reduce subjective feelings of tiredness or fatigue, especially after exercise, may also be related to changes in adrenal steroid regulation. Cortisol counteracts the action of insulin by increasing gluconeogenesis, promoting lipolysis and mobilizing extrahepatic amino acids and ketone bodies, which leads to increased circulating glucose concentrations in the blood (Freeman et al., 2004) and prolonged cortisol secretion causes hyperglycemia (Barseghian et al., 1982). LBP has been reported to enhance the storage of muscle and liver glycogen, to increase the activity of lactate dehydrogenase (LDH) before and after swimming, to inhibit the increase of blood urea nitrogen (BUN) after strenuous exercise, to accelerated the clearance of BUN after exercise, to increase adaptability to an exercise load, to enhance resistance to fatigue and to accelerate the elimination of lactic acid in mice (Luo et al., 2000). However, we found that plasma lactic acid and glucose concentrations were not influenced by Lycium barbarum intake. Nonetheless, participants reported an increase in endurance/energy in the daytime and reduced fatigue following 30 days of Lycium barbarum consumption. These effects were consistent with previous study results (Amagase et al., 2008, 2009a). There is evidence that elevated endogenous glucocorticoid activity can be associated with visceral obesity. This may be mediated centrally level via the HPA-axis and peripherally via increased conversion of cortisone to cortisol by 11-ß-hydroxysteroid-dehydrogenase type 1 in adipose tissue (Masuzaki 2001; Pasquali et al., 1993). Increased activity of the HPA-axis has been linked to metabolic syndrome or "Syndrome X", and may contribute to the clustering of low HDL cholesterol, high triglycerides, insulin resistance, hypertension, and visceral obesity that characterize this syndrome, all of which represent major risk factors for cardiovascular disease, stroke, and diabetes mellitus type II (Brunner, 2002; Brotman et al., 2003). In female weanling mice, LBP was shown to enhance food conversion rate and to reduce body weight after 21 days of consumption (Zhang et al., 2002). We reported a similar reduction in weight gain in adult male rats given Lycium barbarum (Nance et al., 2009). These results suggest that LBP may modulate metabolism in vivo. In support, it was shown in a clinical trial that Lycium barbarum intake increased energy expenditure and/or metabolic rate in humans (Amagase, 2011b). The magnitude of the experimental effects of Lycium barbarum in the current study may in part reflect the fact that the test participants had normal body weights and BMIs in addition to the limited consumption time period. It is possible that the metabolic impact of Lycium barbarum juice would be more readily demonstrated in overweight or obese participants and could be utilized to prevent or treat metabolic syndromes, including glucocorticoid-related conditions. In support of this possibility, animal studies show dramatic effects of $L$ barbarum on blood glucose and insulin levels in diabetic rats that are not observed in nondiabetic controls (Zhao et al., 2005). 
In support of a role for the anti-oxidant properties of Lycium barbarum (Amagase et al., 2009b; Wu et al., 2004; Gong et al., 2005) in the attenuation of the effects of exercise on cortisol release are the results of Davison, et al (2007). Similar to the current results, they showed that dietary supplementation with anti-oxidants (daily vitamin $\mathrm{C}$ (L-ascorbic acid, $1000 \mathrm{mg}$ ) and vitamin E (RRR-alpha-tocopherol, $400 \mathrm{IU})$ supplementation) blunted the cortisol response to a single prolonged exercise challenge (Davison et al., 2007). Also, LBP has been shown to prevent oxidative stress following exhaustive exercise in rats (Shan et al., 2011) and the anti-inflammatory effect of Lycium barbarum consumption was demonstrated by Reeve et al (2010) in an animal model of UV radiation induced oxidative skin damage.

\section{Conclusion}

This is the first randomized trial to evaluate the effects of Lycium barbarum on exercise-induced adrenal steroid in humans. Lycium barbarum intake significantly attenuated the increases in plasma DHEA and cortisol concentrations produced by a short and intense exercise challenge. The Lycium barbarum group showed significant reductions in feelings of tiredness after exercise. Our results suggest that daily consumption of Lycium barbarum may attenuate stress-related reactivity and facilitate adaptation to physical stressors. Furthermore, as elevated endogenous glucocorticoid activity has been linked with visceral obesity and the metabolic syndrome, our findings suggest that altered HPA activity may be related to previously reported metabolic effects of Lycium barbarum and its active ingredients.

\section{Acknowledgements}

The corresponding author (HA) is an employee of FreeLife International. Co-author (DMN) is a member of FreeLife's Independent Scientific Advisory Board.

\section{References}

Amagase, H., Sun, B., \& Borek, C. (2009b). Lycium barbarum (goji) juice shows significant in vivo antioxidant effects in human serum in a randomized, double-blind, placebo-controlled clinical study. Nutrition and Research, 29, 19-25. http://dx.doi.org/10.1016/j.nutres.2008.11.005

Amagase, H., Sun, B., \& Nance, D. M. (2009a). Immunomodulatory Effects of a Standardized Lycium barbarum Fruit Juice in Chinese Older Healthy Human Subjects. Journal of Medicinal Foods, 12(5), 1159-1165. http://dx.doi.org/10.1089/jmf.2008.0300

Amagase, H., \& Farnsworth, N. R. (2011a). A Review of Botanical Characteristics, Phytochemistry, Clinical Relevance in Efficacy and Safety of Lycium barbarum Fruit (Goji). Food Research International, 44, 1702-1717. http://dx.doi.org/10.1016/j.foodres.2011.03.027

Amagase, H., \& Nance, D. M. (2008). A Randomized, Double-blind, Placebo-controlled, clinical study of the general effects of a standardized Lycium barbarum (Goji) Juice, GoChi ${ }^{\mathrm{TM}}$. Journal of Alternative and Complementary Medicine, 14, 403-412. http://dx.doi.org/10.1089/acm.2008.0004

Amagase, H., \& Nance, D. M. (2011b). Lycium barbarum increases caloric expenditure and decreases waist circumference in healthy overweight men and women: Pilot Study. Journal of the American College of Nutrition, 30(5), 304-309.

American Heart Association. (2009). Avnline] Available: http://www.americanheart.org/presenter.jhtml?identifier=3057643 (January 25, 2009)

American Heart Association. (2011). Annline] Avalable: http://www.heart.org/HEARTORG/Conditions/HighBloodPressure/PreventionTreatmentofHighBloodPressure/P hysical-Activity-and-Blood-Pressure_UCM_301882_Article.jsp (December 13, 2011)

Barseghian, G., Rachmiel, L., \& Epps, P. (1982). Direct Effect of Cortisol and Cortisone on Insulin and Glucagon Secretion. Endocrinology, 111, 1648. http://dx.doi.org/10.1210/endo-111-5-1648

Bensky, D., \& Gamble, A. (1993). Gou Qi Zi. In Chinese Herbal Medicine, Materia Medica. (revised ed.), pp. 333-334. Seattle: Eastland Press, Inc.

Brotman, D. J., Girod, J. P., Tankó, L. B., Bagger, Y. Z., Alexandersen, P., Christiansen, C., \& Larsen, P. J. (2003). Can glucocorticoid homeostasis explain the anti-atherogenic effect of peripheral adiposity? Reply. Circulation, 108, e61. http://dx.doi.org/10.1161/01.CIR.0000086784.14560.C3

Brunner, E. J., Hemingway, H., Walker, B. R., et al. (2002). Adrenocortical, autonomic, and inflammatory causes of the metabolic syndrome: nested case-control study. Circulation, 106(21), 2659-2665. http://dx.doi.org/10.1161/01.CIR.0000038364.26310.BD

Buford, T. W., \& Willoughby, D. S. (2008). Impact of DHEA(S) and cortisol on immune function in aging: a brief review. Applied Physiology, Nutrtion, and Metabolism, 33, 429-33. http://dx.doi.org/10.1139/H08-013 
Chang, H. M., \& But, P. P. H. (2001). Gouqizi. In Pharmacology and Applications of Chinese Materia Medica, 2, pp. 852-4. Singapore: World Scientific.

Chang, R. C., \& So, K. F. (2008). Use of anti-aging herbal medicine, Lycium barbarum, against aging-associated diseases. What do we know so far? Cellular and Molecular Neurobiology, 28, 643-52. http://dx.doi.org/10.1007/s10571-007-9181-x

Copeland, J. L., Consitt, L. A., \& Tremblay, M. S. (2002). Hormonal responses to endurance and resistance exercise in females aged 19-69 years. Journals of Gerontology Series A: Biological Sciences and Medical Sciences, 57, B158-65. http://dx.doi.org/10.1093/gerona/57.4.B158

Davison, G., Gleeson, M., \& Phillips, S. (2007). Antioxidant supplementation and immunoendocrine responses to prolonged exercise. Medicine \& Science in Sports \& Exercise, 39, 645-652. http://dx.doi.org/10.1249/mss.0b013e318031303d

Dillon, J. S. (2005). Dehydroepiandrosterone, dehydroepiandrosterone sulfate and related steroids: their role in inflammatory, allergic and immunological disorders. Inflammation \& Allergy - Drug Targets, 4, 377-85. http://dx.doi.org/10.2174/1568010054022079

Filaire, E., Duché, P., \& Lac, G. (1998). Effects of amount of training on the saliva concentrations of cortisol, dehydroepiandrosterone and on the dehydroepiandrosterone: cortisol concentration ratio in women over 16 weeks of training. European journal of applied physiology and occupational physiology, 78, 466-71. http://dx.doi.org/10.1007/s004210050447

Freeman, \& Scott. (2004). Biological Science. (2nd Pkg ed.) Prentice Hall; Upper Saddle River, New Jersey, ISBN 0-13-218746-9.

Gong, H., Shen, P., Jin, L., Xing, C., \& Tang, F. (2005). Therapeutic effects of Lycium barbarum polysaccharide (LBP) on irradiation or chemotherapy-induced myelosuppressive mice. Cancer Biotherapy and Radiopharmacology, 20, 155-62. http://dx.doi.org/10.1089/cbr.2005.20.155

Luo, Q., Yan, J., \& Zhang, S. (2000). Isolation and purification of Lycium barbarum polysaccharides and its antifatigue effect. Wei Sheng Yan Jiu (Journal of Hygiene Research), 29, 115-7.

Mastorakos, G. Paylatou, M. Diamanti-Kandarakis, E., \& Chrousos, G. P. (2005). Exercise and the Stress System. Hormones, 4(2), 73-89.

Masuzaki, H., Paterson, J., Shinyama, H., Morton, N. M., Mullins, J. J., Seckl, J. R., \& Flier, J. S. (2001). A transgenic model of visceral obesity and the metabolic syndrome. Science, 294, 2166-2170. http://dx.doi.org/10.1126/science.1066285

Nance, D. M., Amagase, H., \& Luczy-Bachman, G. (2009). Effects of Lycium barbarum on basal and lps-induced cytokine production. Brain, Behavior, and Immunity, 23(Suppl 2), S51. PNIRS 2009, PsychoNeuroImmunology Research Society Annual Meeting 2009.

Pasquali, R., Cantobelli, S., Casimirri, F., et al. (1993). The hypothalamic-pituitary-adrenal axis in obese women with different patterns of body fat distribution. Journal of Clinical Endocrinology \& Metabolism, 77, 341-346. http://dx.doi.org/10.1210/jc.77.2.341

Radom-Aizik, S., Zaldivar, F. Jr,, Leu, S. Y., Galassetti, P., \& Cooper, D. M. (2008). Effects of 30 min of aerobic exercise on gene expression in human neutrophils. Journal of Applied Physiology, 104, 236-243. http://dx.doi.org/10.1152/japplphysiol.00872.2007

Radom-Aizik, S., Zaldivar, F. Jr., Leu, S. Y., \& Cooper, D. M. (2009). Brief bout of exercise alters gene expression in peripheral blood mononuclear cells of early- and late-pubertal males. Pediatric Research, 65(4), 447-52.

Reeve, V. E., Allanson, M., Arun, S. J., Domanski, D., \& Painter, N. (2010). Mice drinking goji berry juice (Lycium barbarum) are protected from UV radiation-induced skin damage via antioxidant pathways. Photochemical \& Photobiological Sciences, 9, 601-607. http://dx.doi.org/10.1039/b9pp00177h

Shan, X., Zhou J., Ma T., \& Chai Q. (2011). Lycium barbarum Polysaccharides reduce exercise-induced oxidative stress. International Journal of Molecular Sciences, 12, 1081-1088. http://dx.doi.org/10.3390/ijms12021081

Sharkey, B. J., \& Gaskill, S. E. (2007). Fitness \& Health, (six ed.). (pp 55-56, and pp. 100-105). Champaign, IL: Human Kinetics.

Tissandier O., Péres, G., Fiet, J., \& Piette, F. (2001). Testosterone, dehydroepiandrosterone, insulin-like growth factor 1, and insulin in sedentary and physically trained aged men. European Journal of Applied Physiology, 85(1-2), 177-84. http://dx.doi.org/10.1007/s004210100420

Walker, B. R. (2007). Glucocorticoids and cardiovascular disease. European Journal of Endocrinology, 157. 


\section{5-59. http://dx.doi.org/10.1530/EJE-07-0455}

Wu, S. J., Ng, L. T., \& Lin, C. C. (2004). Antioxidant activities of some common ingredients of traditional Chinese medicine, Angelica sinensis, Lycium barbarum and Poria cocos. Phytotheraoy Research, 18, $1008-12$. http://dx.doi.org/10.1002/ptr.1617

Yu, M. S., Lai, C. S., Ho, Y. S., Zee, S. Y., So, K. F., Yuen, W. H., \& Chang, R. C. (2007). Characterization of the effects of anti-aging medicine Fructus lycii on beta-amyloid peptide neurotoxicity. International Journal of Molecular medicine, 20, 261-8.

Zhang, M., Wang, J., \& Zhang, S. (2002). Study on the composition of Lycium barbarum polysaccharides and its effects on the growth of weanling mice. Wei Sheng Yan Jiu (Journal of Hygiene Research), 31, 118-9.

Zhao, R., Qingwang, L. I., \& Xiao, B. (2005). Effects of Lycium barbarum plysaccharide on the improvement of insulin resistance in NIDDM rats. Yakugaku Zasshi, 125, 981-8. http://dx.doi.org/10.1248/yakushi.125.981

Abbreviations: DHEA, dehydroepiandrosterone; ACTH, adrenocorticotropic hormone; L. barbarum, Lycium barbarum; LBP, Lycium barbarum polysaccharide; IL, interleukin; WR, work rate; ELISA, Enzyme-Linked Immuno Sorbent Assay; BMI, body mass index; LDH, lactate dehydrogenase; BUN, blood urea nitrogen; RMR, resting metabolic rate; HPA, hypothalamic-pituitary-adrenal.

Table 1. (A) Baseline demographic variables of non-discriminated participants in the current randomized, double-blind, placebo-controlled human clinical study. (B) Parameters of maximal heart rate, watts and calorie burned during the short and intense exercise challenge at pre- and post-intervention in both Lycium barbarum (L. barbarum) and placebo groups. This exercise challenge was performed only on Day 1 (pre-intervention) and Day 30 (post-intervention) as physical stress.

A

\begin{tabular}{|c|c|c|c|c|}
\hline & Variable & & $\mathbf{n}$ & Mean \pm SEM \\
\hline \multirow{2}{*}{ Age (years old) } & & Placebo & 19 & $31.1 \pm 2.5$ \\
\hline & & L. barbarum & 20 & $36.0 \pm 2.9$ \\
\hline \multirow{2}{*}{ Female Population (\%) } & & Placebo & 19 & 63.2 \\
\hline & & L. barbarum & 20 & 70.0 \\
\hline \multirow{2}{*}{ Exercise (times/week) } & & Placebo & 19 & $0.5 \pm 0.1$ \\
\hline & & L. barbarum & 19 & $0.3 \pm 0.1$ \\
\hline \multirow{4}{*}{$\begin{array}{l}\text { Average Daily Caloric } \\
\text { Intake (kcal)* }\end{array}$} & \multirow{2}{*}{ Pre-intervention/wash-out period } & Placebo & 19 & $1,866 \pm 158$ \\
\hline & & L. barbarum & 20 & $1,972 \pm 181$ \\
\hline & \multirow{2}{*}{$\begin{array}{l}\text { during the } 30 \text {-day intervention } \\
\text { study period }\end{array}$} & Placebo & 19 & $1,807 \pm 134$ \\
\hline & & L. barbarum & 20 & $1,839 \pm 144$ \\
\hline
\end{tabular}

Each value indicates mean \pm SEM except female population. Sample numbers varied due to missing answers. *calculated from daily food diary. Age and average daily caloric intake data were analyzed by ANOVA, and exercise frequency data were analyzed with nonparametric Mann-Whitney U Test (Placebo vs Lycium barbarum). There was no statistical significance between the groups.

B

\begin{tabular}{lccrrr}
\hline & & n & Pre-intervention & n & Post-intervention \\
\hline \multirow{2}{*}{ Heart Rate (beat/min) } & Placebo & 19 & $139.1 \pm 3.0$ & 19 & $145.3 \pm 3.2$ \\
& L. barbarum & 20 & $141.1 \pm 2.7$ & 20 & $138.8 \pm 2.7$ \\
\hline \multirow{2}{*}{ Total Watts } & Placebo & 19 & $82.3 \pm 5.6$ & 19 & $98.9 \pm 8.1$ \\
& L. barbarum & 20 & $79.8 \pm 4.5$ & 20 & $91.2 \pm 5.2$ \\
\hline \multirow{2}{*}{ Calorie burned (kcal) } & Placebo & 19 & $204.7 \pm 11.2$ & 19 & $223.6 \pm 17.1$ \\
& $L$ barbarum & 20 & $190.7 \pm 8.1$ & 20 & $207.4 \pm 11.9$
\end{tabular}

Each value indicates mean \pm SEM. N.S., not significant analyzed by ANOVA. There was no statistical significance between the groups, or pre- and post-intervention. 
Table 2. Effect of Lycium barbarum (L. barbarum) or placebo on (A) anthropometric parameters and (B) subjective indications compared to pre-intervention in a randomized, double-blind, placebo-controlled human clinical study

$\mathbf{A}$

\begin{tabular}{|c|c|c|c|c|c|}
\hline & & $\mathbf{N}$ & Pre-intervention & $\mathbf{n}$ & Post-intervention \\
\hline \multirow{2}{*}{ Body weight (kg) } & Placebo & 19 & $82.3 \pm 5.1$ & 19 & $82.5 \pm 5.1$ \\
\hline & L. barbarum & 20 & $82.4 \pm 4.8$ & 20 & $82.7 \pm 5.0$ \\
\hline \multirow{2}{*}{$\begin{array}{l}\text { Body mass index }(\mathrm{BMI}) \\
\left(\mathrm{kg} / \mathrm{m}^{2}\right)\end{array}$} & Placebo & 19 & $29.3 \pm 1.7$ & 19 & $29.4 \pm 1.7$ \\
\hline & L. barbarum & 20 & $29.5 \pm 1.6$ & 20 & $29.7 \pm 1.6$ \\
\hline \multicolumn{6}{|l|}{$\overline{\mathbf{B}}$} \\
\hline \multirow{2}{*}{ Tiredness } & Placebo & 19 & $2.2 \pm 0.3$ & 19 & $1.8 \pm 0.4$ \\
\hline & L. barbarum & 20 & $2.4 \pm 0.4$ & 19 & $1.9 \pm 0.4$ \\
\hline \multirow{2}{*}{ Muscular complaints } & Placebo & 18 & $1.9 \pm 0.3$ & 19 & $1.9 \pm 0.3$ \\
\hline & L. barbarum & 18 & $1.9 \pm 0.3$ & 20 & $1.8 \pm 0.3$ \\
\hline \multirow{2}{*}{ Physical discomfort } & Placebo & 19 & $1.7 \pm 0.3$ & 19 & $1.4 \pm 0.3$ \\
\hline & L. barbarum & 20 & $1.9 \pm 0.4$ & 20 & $1.6 \pm 0.4$ \\
\hline \multirow{2}{*}{ Joint pain } & Placebo & 19 & $1.4 \pm 0.3$ & 19 & $1.6 \pm 0.4$ \\
\hline & L. barbarum & 19 & $2.1 \pm 0.4$ & 19 & $1.8 \pm 0.4$ \\
\hline \multirow{2}{*}{ Stiff Shoulder } & Placebo & 19 & $1.6 \pm 0.4$ & 19 & $1.7 \pm 0.4$ \\
\hline & L. barbarum & 20 & $2.4 \pm 0.4$ & 20 & $2.1 \pm 0.4$ \\
\hline \multirow{2}{*}{ Tiredness after exercise } & Placebo & 19 & $1.7 \pm 0.3$ & 19 & $1.9 \pm 0.3$ \\
\hline & L. barbarum & 20 & $2.1 \pm 0.3$ & 20 & $1.4 \pm 0.2^{a}$ \\
\hline \multirow{2}{*}{ Feeling of bad circulation } & Placebo & 19 & $0.1 \pm 0.1$ & 19 & $0.3 \pm 0.1$ \\
\hline & L. barbarum & 20 & $1.3 \pm 0.4$ & 20 & $0.5 \pm 0.1^{a}$ \\
\hline Overall health conditions & Placebo & 19 & $60.0 \pm 7.0$ & 19 & $59.9 \pm 8.5$ \\
\hline (Total Scores) & L. barbarum & 20 & $79.2 \pm 8.3$ & 20 & $67.3 \pm 8.7^{a}$ \\
\hline
\end{tabular}

Each value indicates mean \pm SEM. Anthropometric parameters were analyzed by ANOVA. These subjective indications were collected from the questionnaire asked immediately before the short and intense exercise challenge on both pre- (Day 1) and post-intervention (Day 30). Sample numbers varied due to missing answers. Lower scores reflect improvements in subjective ratings. ${ }^{a}$ indicates significant difference $(\mathrm{P}<0.05)$ from pre-intervention analyzed by the nonparametric Wilcoxon matched pairs tests. 
Table 3. Effect of Lycium barbarum (L. barbarum) or placebo on plasma dehydroepiandrosterone (DHEA), cortisol, lactic acid, glucose and blood urea nitrogen (BUN) concentrations at pre- and post-exercise in a randomized, double-blind, placebo-controlled human clinical study

\begin{tabular}{|c|c|c|c|c|c|c|c|c|c|}
\hline & & \multicolumn{4}{|c|}{ Pre-intervention } & \multicolumn{4}{|c|}{ Post-intervention } \\
\hline & & $\mathbf{n}$ & Pre-exercise & $\mathbf{n}$ & Post-exercise & $\mathbf{n}$ & Pre-exercise & $\mathbf{n}$ & Post-exercise \\
\hline \multirow{2}{*}{$\begin{array}{l}\text { DHEA } \\
(\mu \mathrm{g} / \mathrm{dl})\end{array}$} & Placebo & 18 & $8.2 \pm 1.1$ & 18 & $10.4 \pm 1.2^{b}$ & 18 & $7.3 \pm 0.8$ & 18 & $11.5 \pm 1.0^{b}$ \\
\hline & $\begin{array}{l}L . \\
\text { barbarum }\end{array}$ & 17 & $6.1 \pm 1.1$ & 18 & $8.4 \pm 1.0^{b}$ & 18 & $5.2 \pm 0.9$ & 18 & $5.7 \pm 0.9$ \\
\hline \multirow{2}{*}{$\begin{array}{l}\text { Cortisol } \\
(\mu \mathrm{g} / \mathrm{dl})\end{array}$} & Placebo & 18 & $34.8 \pm 6.2$ & 18 & $52.2 \pm 12.4^{b}$ & 18 & $23.0 \pm 2.1$ & 18 & $51.8 \pm 15.5^{b}$ \\
\hline & $\begin{array}{l}L . \\
\text { barbarum }\end{array}$ & 15 & $37.3 \pm 4.3$ & 18 & $47.6 \pm 6.8$ & 18 & $47.8 \pm 11.1$ & 18 & $42.4 \pm 4.3$ \\
\hline \multirow{2}{*}{$\begin{array}{l}\text { Lactic } \\
\text { acid } \\
(\mathrm{mmol} / \mathrm{l})\end{array}$} & Placebo & 18 & $2.5 \pm 0.2$ & 18 & $6.1 \pm 0.5^{b}$ & 18 & $2.3 \pm 0.3$ & 18 & $6.1 \pm 0.5^{b}$ \\
\hline & $\begin{array}{l}L . \\
\text { barbarum }\end{array}$ & 18 & $2.4 \pm 0.2$ & 18 & $5.8 \pm 0.4^{b}$ & 18 & $2.2 \pm 0.2$ & 18 & $5.8 \pm 0.5^{b}$ \\
\hline \multirow{2}{*}{$\begin{array}{l}\text { Glucose } \\
(\mathrm{mg} / \mathrm{dl})\end{array}$} & Placebo & 19 & $91.3 \pm 2.1$ & 19 & $90.8 \pm 2.8$ & 19 & $90.1 \pm 1.8$ & 19 & $92.5 \pm 3.2$ \\
\hline & $\begin{array}{l}L . \\
\text { barbarum }\end{array}$ & 20 & $99.2 \pm 10.3$ & 20 & $95.3 \pm 7.8$ & 20 & $98.9 \pm 9.5$ & 20 & $96.1 \pm 7.2$ \\
\hline \multirow{2}{*}{$\begin{array}{l}\text { BUN } \\
(\mathrm{mg} / \mathrm{dl})\end{array}$} & Placebo & 19 & $12.1 \pm 0.9$ & 19 & $12.4 \pm 0.8$ & 19 & $13.5 \pm 1.0^{a}$ & 19 & $13.7 \pm 0.9^{a}$ \\
\hline & $\begin{array}{l}\text { L. } \\
\text { barbarum }\end{array}$ & 20 & $11.7 \pm 0.6$ & 20 & $11.9 \pm 0.6$ & 20 & $13.5 \pm 0.6^{a}$ & 20 & $13.9 \pm 0.6^{a}$ \\
\hline
\end{tabular}

An exercise challenge on a cycle ergometer at $70 \%$ of age-adjusted maximum heart rate was given on each individual participant at pre- (Day 1) and post-intervention (Day 30). Parameters of maximal heart rate, watts and calorie burned during the exercise challenge were not statistically different in both pre- and post-intervention and also in both Lycium barbarum and placebo groups. This exercise challenge was performed only at the time of pre- and post-intervention. Intervention period of Lycium barbarum or placebo was 30 days. Each value indicates mean \pm SEM. Sample numbers varied due to missing samples. ${ }^{a},{ }^{b}$ indicate significant difference $(\mathrm{P}<0.05)$ from pre-intervention and pre-exercise, respectively analyzed by ANOVA. 


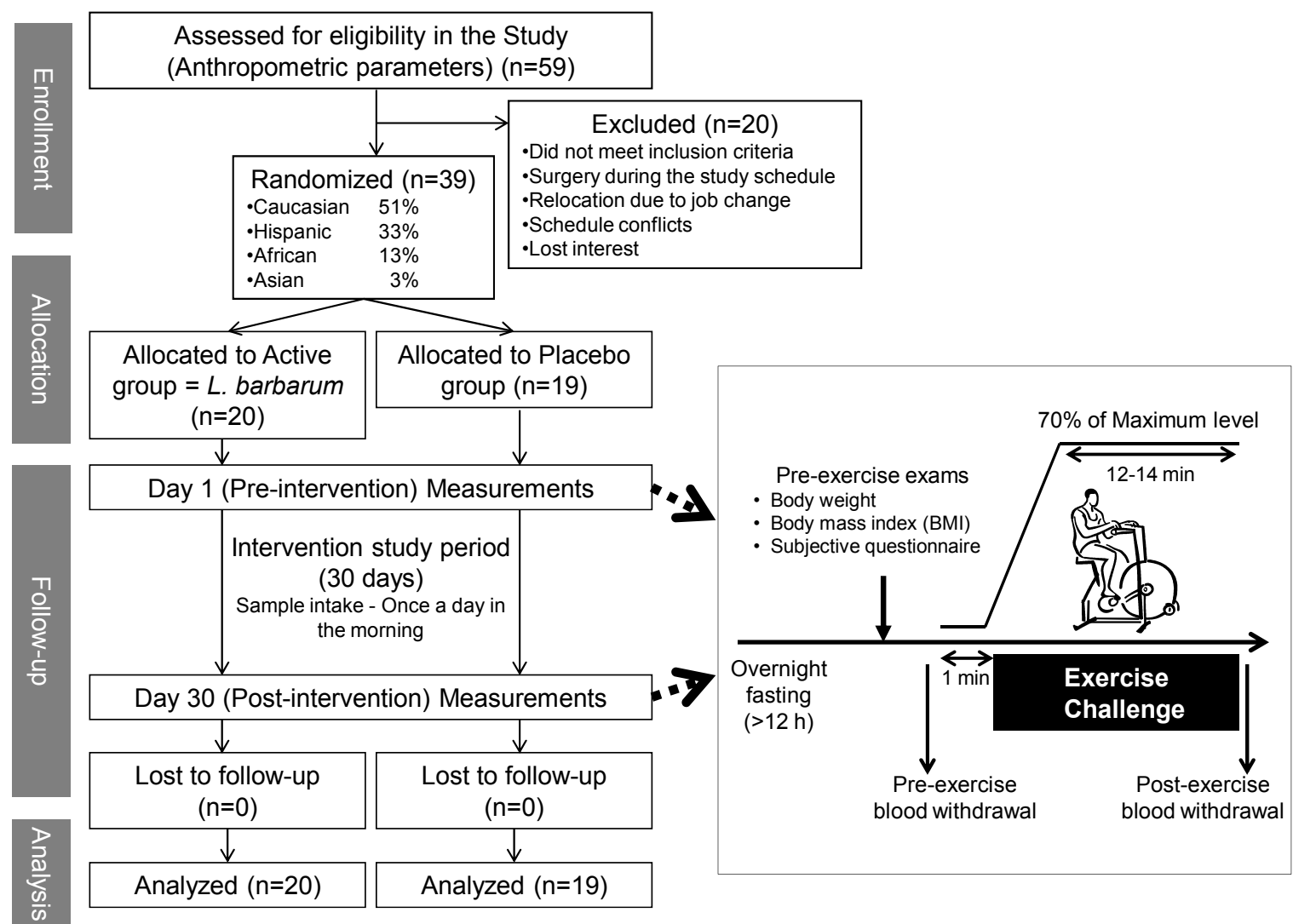

Figure 1. CONSORT flow diagram, study design and the experimental block on Day 1 (pre-intervention) and Day 30 (post-intervention). Participants completed pre-exercise examinations including anthropometric parameter measurements, subjective questionnaire in about $15 \mathrm{~min}$, followed by a pre-exercise blood withdrawal, a short and intense exercise challenge on a cycle ergometer at $70 \%$ of age-adjusted maximum heart rate by loading ride for about 12-14 min and post-exercise blood withdrawal on both Day 1 and Day 30 